CYBERNETICS AND INFORMATION TECHNOLOGIES • Volume 16, No 6

Special issue with selection of extended papers from 6th International Conference on Logistic, Informatics and Service Science LISS'2016

Sofia $\bullet 2016$

\title{
Low Cost Locating Method of Wireless Sensor Network in Precision Agriculture
}

\section{Cunjiang $Y u$}

Changchun University, Changchun, China

Email:35821940@qq.com

Abstract: The wireless sensor network covers more scale with more sensor nodes for larger scale agriculture. The article describes improvement of DV-Hop Algorithm to locate the nodes with quadrilateral range positioning method, so that the difficulty of dilatation method in agriculture actual application to be solved. The analog test for the algorithm is conducted and is mainly developed for the average locating error with illustration and discussion on the proportion relations of average error, average connectivity and anchor nodes. According to the analog results, the algorithm obtains better effect on the average locating error, which improves the accuracy of the algorithm.

Keywords: Wireless sensor network, agriculture, low cost location, DV-Hop.

\section{Introduction}

In the sensor network, the location information is very important for the sensor network monitoring, related to the information transmission of the overall network. If it fails to obtain the node locations, the monitoring function of the sensor network will be useless. Since the agriculture WSN node locations are provided with much change, limits of cost, power consumption and hardware constraints of the wireless sensor network, so one of the important research focuses is the achievement of low cost, low power consumption and higher accuracy location for agriculture's large scale wireless sensor network system.

Data is the key point in application of the wireless sensor network. While considering the data, the location information shall be defined in acquisition of nodes data, since the monitoring information without location information is meaningless [1]. It is one of the basic functions of the wireless sensor network to define the occurred event location or nodes information of data acquisition. In order to supply effective location information, the sensor nodes shall be provided with function of confirming the location by themselves after the network establishment in the case of random disposition of sensor nodes. 


\section{Locating technology}

According to the sensor network nodes locating technology, the sensor nodes can be specified as beacon nodes and unknown nodes according to whether the node location is confirmed by itself. Generally, only small amount of nodes are capable of confirming the locations, such as devices obtained by GPS receiver or erected on the appointed locations manually, and they are defined as beacon nodes. But most of nodes are unknown, connected into the network by random disposition due to limits of application, power consumption, etc. in actual application, which results to unknown locations. The sensor network locating technology has to estimate the unknown node locations with the help of the information of small amount beacon nodes, which estimation is conducted by measuring the ranges, angles, connection information, topology relation of neighbored nodes.

Neighbored nodes: The other nodes within communication radius of sensor nodes. If two nodes can be in communication with each other, the two nodes can be defined as neighbored nodes to each other.

Hop number: The total amount of hops within the range of two nodes; the hop number between the two neighbored nodes is 1 . If $\mathrm{A}$ is neighbored with $\mathrm{B}$, and $\mathrm{B}$ is neighbored with $\mathrm{C}$, but $\mathrm{C}$ and $\mathrm{A}$ are not neighbored, the hop number between $\mathrm{A}$ and $\mathrm{C}$ is $2, \mathrm{~A}$ and $\mathrm{C}$ can be defined as 2 hops neighbor to each other. Therefore, $k$ hops conception is provided $(k=1,2, \ldots)$.

Reference nodes: The conception used in locating calculation. Generally, while conducting the estimation for unknown nodes location, the range equation or geometric constraint conditions established by some beacon nodes shall be used. The selected reference node collections by unknown nodes are different in uniform locating algorithm [2].

Arrival time: The transmission time from one node to another.

Difference of arrival times: It can be specified as two cases. Firstly, the transmission time difference of two signals from one node to another with different transmission speed. Secondly, the transmission time difference of two nodes sending the same kind of signals to one receiving node.

Receiving signal intensity: Intensity of received wireless signal by nodes.

Arrival angle: The angle of the node signal receiving direction and self-axial line.

\section{Improved DV-Hop Algorithm}

The locating algorithm can be classified by some various classification modes in the sensor network. The range-based and range-free algorithms can be classified according to whether the ranges among the nodes are measured. Generally, the range-based method includes three procedures: firstly, to measure the range or angle from the unknown node to the neighbored reference node, and continue to calculate the range or location to the beacon node; secondly, to conduct basic dilatation method to estimate the initial location of the common node by using the reference node location and corresponding range or angle information; finally, to 
conduct iteration calculation for the estimated location of the common node by taking certain measures [4].

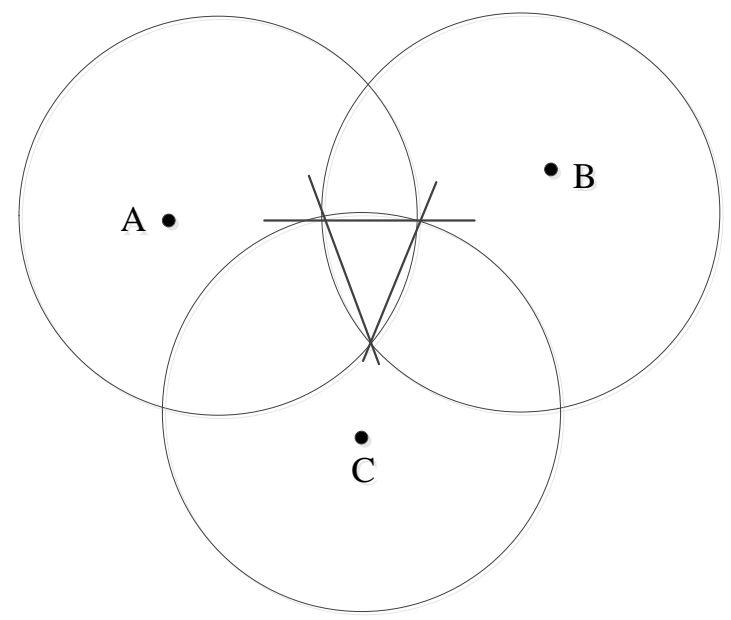

Fig. 1. Actual situation of trilateration method

There are four common algorithms that can be applied to the sensor network location, including TOA-based location, TDOA-based location, AOA-based location and RSSI-based location. Three common measuring technologies are used, which includes arrival time, arrival angle and signal attenuation. The arrival time can be classified as arrival time of TOA and arrival time difference of TDOA. TOA means that the signal transmission speed is known, so that the range from the signal sending node to the receiving node can be estimated by using the transmission time. TDOA includes two modes, firstly, the transmission time difference of two signals from one node to another one with different transmission speed can be used to estimate the range; secondly, the transmission time difference of two nodes sending the same kind of signals to one receiving node can be used to estimate the range difference to the two sending nodes. There are many location methods based on TOA or TDOA. Arrival angle of AOA means the angle between the node signal receiving direction and self axial line. There are many location methods based on AOA as well. For RSSI location, the node sending signal intensity is known, the receiving node shall estimate the range according to the intensity of received signal. A great many of studies have been done based on RSSI location method [6].

\subsection{Conventional DV-Hop Algorithm}

With the range-free location technology is no need of measuring the ranges or location information among nodes; the hardware requirements are greatly reduced. At present, there are two main range-free location methods. One method is to estimate the range from the unknown node to the beacon node, and then conduct locating by using dilatation method or maximum likelihood estimation method; another method is to confirm the area including the unknown nodes with the help of neighbored nodes and beacon nodes, and then take the centre of the area as the 
location of the unknown node. The accuracy of range-free location is insufficient, therefore, the accuracy improvement is required.

The analysis of wireless radio-frequency attenuation and drop-out rate shall be conducted in $4333 \mathrm{MHz}$ wireless channel by using empirical model in different transmission ranges and aerial heights, different crop heights and densities, and different terrains; then the connectivity of information transmission shall be calculated as follows:

$$
\mathrm{CM}_{k, a_{i}}=\frac{n_{\text {recv }}\left(a_{i}, t\right)}{n_{\text {send }}\left(a_{i}, t\right)} 100 \%,
$$

$N_{\text {recv }}\left(a_{i}, t\right)$ is in time scale of $t$, the received signal quantity $a_{i}$ by node $k$ from the beacon node; $n_{\text {send }}\left(a_{i}, t\right)$ is the sent signal quantity from anchor node $a_{i}$ in scale of $t$. Let's set the threshold value as $90 \%$, the nodes which connectivity is $\mathrm{CM}_{k_{i} a_{i}}>90 \%$ shall be taken as the neighbored nodes.

For the locating algorithm of DV-Hop, the known node shall calculate the minimum hops to the anchor node, and then estimate the average distance of onehop that shall be multiplied with the minimum hops to obtain the estimated distance from the unknown node to the anchor nodes; finally, the known node location shall be calculated by using dilatation method or maximum likelihood estimation method.

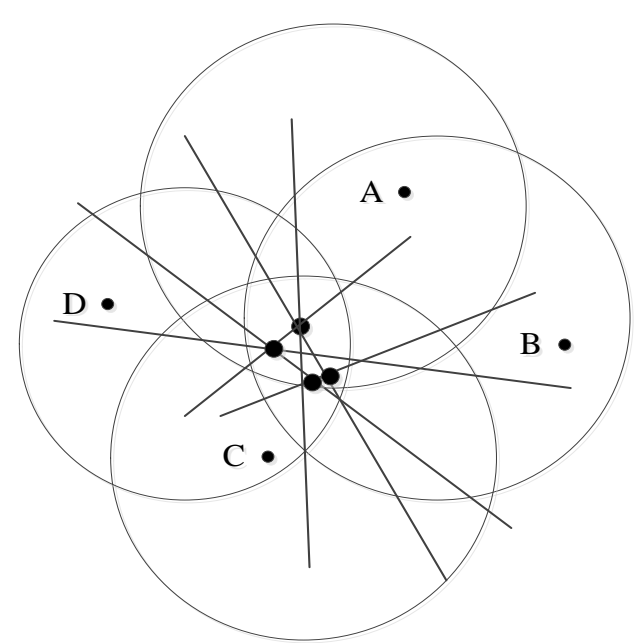

Fig. 2. Actual situation of trilateration method

\subsubsection{Minimum hops calculation for unknown node}

The beacon node broadcasts its location information packet to neighbored nodes; the packet includes hop digital field with initial setting of 0 . The receiving node shall record the minimum hops for each beacon node and, at the same time, neglect the larger hops packet from the same anchor node; then it shall adjust the data to 1 and send it to the neighbored nodes. The minimum hops of all beacon nodes in the network shall be recorded by using this method [8]. 
3.1.2. Calculate the actual one-hop distance of the unknown node and beacon node

Each anchor node shall estimate the average one-hop actual distance by using the next formula according to the other anchor nodes location information and hops recorded in the first stage:

$$
\text { HopSize }_{i}=\frac{\sqrt{\left(x_{i}-x_{j}\right)^{2}+\left(y_{i}-y_{j}\right)^{2}}}{H_{j}} .
$$

In the formula, $\left(x_{i}, y_{i}\right),\left(x_{i}, y_{i}\right)$ are the beacon nodes location of $i$ and $j$, and $H_{j}$ is the hop quantity between beacon nodes $i$ and $j, i \neq j$.

The beacon node shall broadcast the calculated one-hop average distance to the network by using the packet with lifetime field; the unknown node shall only record the value of the first received one-hop average distance and then transmit it to the neighbored nodes; this method can ensure most of nodes to receive the onehop average distance value from the nearest anchor nodes. After receiving average one-hop distance by the known node, the hop distance to each beacon node shall be calculated according to the recorded hops.

3.1.3. Calculate the self location by using dilatation method or maximum likelihood estimation method

The known node shall calculate the self location by using the hop distance to each anchor node recorded in the second stage and by using dilatation method or maximum likelihood estimation method.

\subsection{Improved DV-Hop Algorithm}

DV-Hop Algorithm takes the communication radius of the node as the average onehop distance, so the location error is large. In case of sparse nodes, the hops from the unknown node to the beacon node shall be increased, and the error between the hop distance and actual distance shall be enlarged. The distance from unknown node $D$ illustrated previously in the three sides measuring method to three anchor nodes of $A, B$ and $C$ are separately $d_{A}, d_{B}, d_{C}$. Let's take the error node as the circle center to draw three circles with the distance value; the crossover point of the three circles is the location of the unknown node $D$. With the influence of aerial height, plant variety and intensity, the wireless signal shall be attenuated because of reflection, scattering and absorption during passing the crop in actual complex agriculture environment. Dilatation method produces certain deviation, so that the three circles are incapable of crossing at the same point, which is showed on Fig. 1.

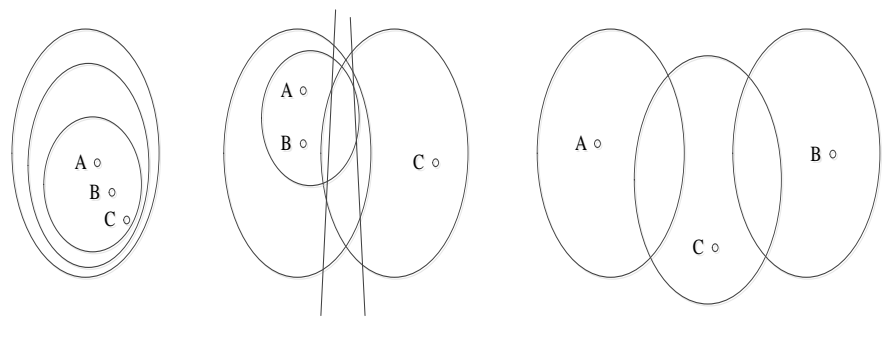

Fig. 3. Error in location information 
In order to solve the problems in actual application and improve the accuracy, each beacon node can obtain the distance $\mathrm{HopSize}_{i}$ of average one-hop according to Formulas (4)-(8) bellow. By using the mode of flooding routine, the unknown node shall obtain the average one-hop distance of each error node, which distance value shall be weighed to obtain the final average one-hop distance.

3.2.1. If the hops from the unknown node to each beacon node are $h_{1}, h_{2}, h_{3}, \ldots, h_{n}$, the weighing value of each error node's average one-hop distance is:

$$
W_{i}=\frac{\frac{1}{h_{i}}}{n_{i=1}^{n}\left(\frac{1}{h_{i}}\right)} .
$$

The final average one-hop distance of the unknown node is recorded as $S$,

$$
S={ }_{i=1}^{n} W_{i} \text { HopSize } i \text {. }
$$

3.2.2. Correction for the distance value

$$
\text { Correction }_{i, j}=\frac{\left(\begin{array}{lll}
H_{i, j} & \operatorname{HopSize}_{i} & \operatorname{Dis}_{i, j}
\end{array}\right)}{\operatorname{Dis}_{i, j}},
$$

$\operatorname{Dis}_{i, j}$ is the actual measured distance from anchor node $i$ and $j$. The formula is as follows: $\operatorname{Dis}_{i, j}=\sqrt{\left(x_{i}-x_{j}\right)^{2}+\left(y_{i}-y_{j}\right)^{2}}$. The product of final average one-hop distance $S$ and the hops $h_{i}$ between the unknown node and the beacon node $i$ shall be taken as the distance from the unknown node to the beacon node $i$; the formula is $\operatorname{dis}_{i}=S \times h_{i}$. The final correction distance after correcting the distance value is as follows:

$$
\operatorname{disCorrk,t}=\frac{\operatorname{dis}_{k, i}}{1+\text { Correction }_{k, i} \quad h_{k, i}} .
$$

Based on the dilatation method, one more error node shall be added, as shown on Fig. 2. According to the principle of the dilatation method, 3 error nodes shall be taken each time to estimate the unknown node location, and four groups of location estimation are obtained from the 4 error nodes. $E$ point location estimation from $B$, $C$ and $D$ is $E_{1}\left(x_{E 1}, y_{E 1}\right) ; E$ point location estimation from $A, B$ and $C$ is $E_{2}\left(x_{E 2}, y_{E 2}\right)$; $E$ point location estimation from $A, B$ and $D$ is $E_{3}\left(x_{E 3}, y_{E 3}\right) ; E$ point location estimation from $A, C$ and $D$ is $E_{4}\left(x_{E 4}, y_{E 4}\right)$; Locations of four anchor nodes are respectively $A\left(x_{A}, y_{A}\right), B\left(x_{B}, y_{B}\right), C\left(x_{C}, y_{C}\right)$ and $D\left(x_{D}, y_{D}\right)$; the distance from the unknown node $E\left(x_{E}, y_{E}\right)$ to each anchor node is respectively $r_{A}, r_{B}, r_{C}, r_{D}$, In order to improve the location accuracy further, the weighted factors, the radiuses summary reciprocal of each time locating three circles are adopted in each group of location coordinates; the calculation formula is as follows: 


$$
\begin{aligned}
& x_{E}=\frac{\frac{x_{E 1}}{r_{B}+r_{C}+r_{D}}+\frac{x_{E 2}}{r_{A}+r_{B}+r_{C}}+\frac{x_{E 3}}{r_{A}+r_{B}+r_{D}}+\frac{x_{E 4}}{r_{A}+r_{C}+r_{D}}}{\frac{1}{r_{A}+r_{B}+r_{C}}+\frac{1}{r_{A}+r_{B}+r_{D}}+\frac{1}{r_{A}+r_{C}+r_{D}}+\frac{1}{r_{B}+r_{C}+r_{D}}}, \\
& y_{E}=\frac{\frac{y_{E 1}}{r_{B}+r_{C}+r_{D}}+\frac{y_{E 2}}{r_{A}+r_{B}+r_{C}}+\frac{y_{E 3}}{r_{A}+r_{B}+r_{D}}+\frac{y_{E 4}}{r_{A}+r_{C}+r_{D}}}{\frac{1}{r_{A}+r_{B}+r_{C}}+\frac{1}{r_{A}+r_{B}+r_{D}}+\frac{1}{r_{A}+r_{C}+r_{D}}+\frac{1}{r_{B}+r_{C}+r_{D}}} .
\end{aligned}
$$

During the locating process, the conditions showed in Fig. 3 shall be eliminated when selecting the locating circles for reference.

\section{Algorithm process}

Step 1. The periodical broadcast of the beacon node includes ID, self location and digital packet of counter information with initial setting of 0 .

Step 2. Determine whether the received digital packet is a beacon node; if yes, the beacon node will receive the broadcast digital packet from another beacon node; else (case of unknown node) the broadcast digital packet shall be transmitted after adding 1 in its counter.
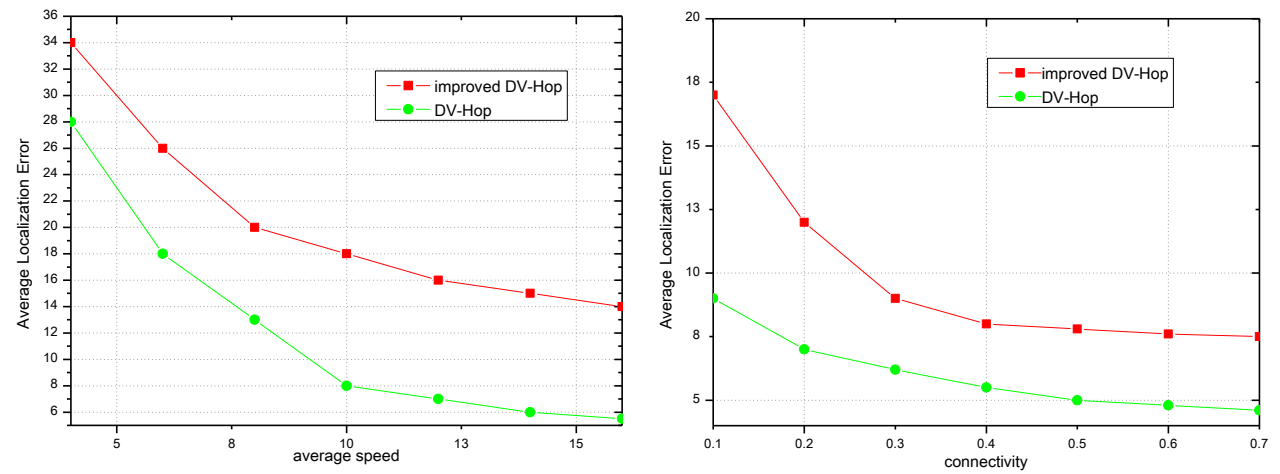

Fig. 4. Simulation result

Step 3. Each beacon node will calculate the distance $\operatorname{Dis}_{i, j}$ to other beacon nodes according to the location information of other beacon nodes' digital packets and self location information, so the estimated average one-hop distance HopSize ${ }_{i}$ of this error node and other error nodes' hops information shall be calculated according to Formula (5).

Step 4. The beacon node $i$ distance value shall be corrected to Correction ${ }_{i, j}$ according to Formulas (4)-(7), which shall be conducted for each beacon node.

Step 5. The broadcast digital packet of the beacon node $i$ includes ID, average one-hop distance of HopSize $i$, correction value of Correction $_{i, j}$ and a counter with initial setting of 0 .

Step 6. Determine whether the unknown node has received the error node's digital packet; if yes, compare the two digital packets and store the packet with lower counter's value; else, store the digital packet of the error node. The unknown node shall store the received error node's digital packet, including the counter's 
value $h_{i}$, average one-hop distance value of $\mathrm{HopSize}_{i}$ and correction value of Correction $_{i, j}$, then transmit the digital packet after adding 1 to the counter.

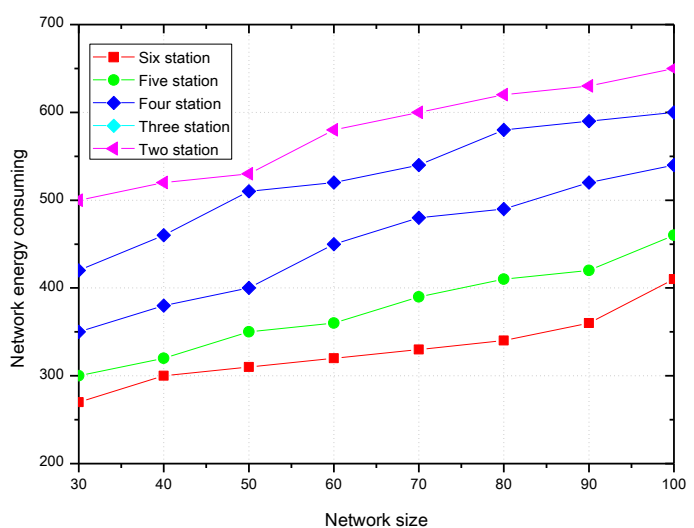

Fig. 5. Network energy consuming

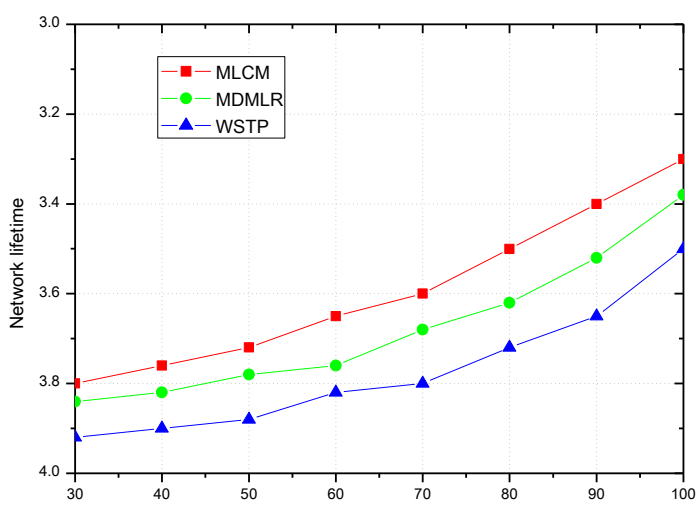

Fig. 6. Comparison of network lifetime

Step 7. Conduct weighing for the average one-hop distance information HopSize $_{i}$ of each beacon node obtained by the unknown node to calculate the final average one-hop distance of the unknown node. According to Formula (2), the unknown node hi is the allocated weighing value of average one-hop distance HopSize $_{i}$. According to Formula (3), calculate the final average one-hop distance $S$ of the unknown node.

Step 8. The distance $L_{i}$ from the unknown node to the error node $i$ is equal to the product of the average one-hop distance $S$ and the hops $h_{i}$ from the unknown node to the beacon node $i$; the formula is $L_{i}=S h_{i}$.

Step 9. Calculate the correction distance from the unknown node to the beacon node.

$$
\operatorname{disCorr}_{k, j}=\frac{L_{i}}{1+\text { Correction }_{i, j} \quad h_{k, i}} .
$$


Step 10. The distance values shall be sequenced from big to small; select any three beacon nodes from the four beacon nodes to locate; the data shall be eliminated in case of the figure until the location requirements are satisfied.

Step 11. According to the dilatation method, four groups of data shall be obtained from four beacon nodes in normal conditions.

Step 12. According to Formula (6), calculate the unknown node coordinates by using the algorithm method of Weighing Centre.

\section{Emulation analysis}

The Jinxing Fengqiao orchard is selected as the test area, and 30 nodes are arranged randomly in the test area with communication radius of $60 \mathrm{~m}$. 100 times of test are conducted for the calculated location information of sensor nodes, the test data has the average value of the 100 times of test. Various algorithms are adopted to compare the measured locating error results in proportion to various connectivity and anchor nodes; the analog result is shown on Fig. 4.

The focus of the emulation is to study and analyze the relation of the reconfiguration error, which is in DCS coding and decoding algorithm based on the time dependency and the quantity of observation data. The emulation adopts Matlab as tool, and the space dependency data source in WSN adopts two-dimension Gaussian distribution to conduct the analog; the nodes of WSN are randomly and evenly distributed within event area of $60 \times 60 \mathrm{~m}$. The relation of the reconfiguration error and the quantity of observation data are studied and analysed. All sensor nodes of WSN produce sensory data at the same time and send it to the cluster head node of the coding end in the system; the cluster head node uses DCS Coding Algorithm to compress and encode the received sensory data; the produced observation data is transmitted to the decoding end through wireless multi-hop mode. Finally, the assembled nodes use DCS Decoding Algorithm to conduct accuracy reconfiguration for the sensory data of each node in WSN event area.

As it is shown on the Fig. 4, the improved DV-Hop obtains better result for the average locating error of the unknown node, so the algorithm is improved.

\section{Conclusion}

The wireless sensor network covers more scale with more sensor nodes for larger scale agriculture. The article describes improvement of DV-Hop Algorithm to locate the nodes with quadrilateral range positioning method, so that the difficulty of dilatation method in agriculture actual application to be solved. The analog test for the algorithm is conducted, and is mainly developed for the average locating error with illustration and discussion on the proportion relations of average error, average connectivity and anchor nodes. According to the analog results, the algorithm obtains better effect on the average locating error, which improves the accuracy of the algorithm. 


\section{References}

1. Nishimura, C. E., D. M. Conlon. Monitoring Whales and Earthquakes Using SOSUS. March Techology Social Journal, Vol. 27, May 2004, pp. 13-21.

2. N a g p a l, R., H. S h r o b e. Organizing a Global Coordinate System from Local Information on an Ad Hoc Sensor Network. - Information Processing in Sensor Networks, Vol. 22, August 2004, pp. 333-348.

3. He id e ma n n, J., E. S i lva. Building Efficient Wireless Sensor Networks with Low Level Naming. - In: Proc. of 18th ACM Symposium on Operating System Principles, Vol. 4, August 2011, pp. 146-151.

4. Guibas, L. J. Tracking, Sensing and Reasoning with Relations. - IEEE Signal Processing Manazine, Vol. 19, February 2002, pp. 73-85.

5. Rabaey, M. J., M. A mmer. Picoradio Supports Ad Hoc Ultra-Low Power Wireless Networking. - IEEE Computer Magazine, Vol. 33, May 2000, pp. 42-48.

6. Warneke, B., M. Last. Communicating with a Cubic-Millimeter Computer. - IEEE Computer, Vol. 34, May 2001, pp. 44-51.

7. Shih, E., C. I ckes n. Physical Layer Driven Protocol and Algorithm Design for EnergyEfficient Wireless Sensor Networks. - In: Proc. of ACM Conference on Mobile Computing and Networking, Vol. 41, pp. 272-287.

8. Ghiasi, S., A. Srivastava. Optimal Energy Aware Clustering in Sensor Networks. Sensors, Vol. 2, August 2002, pp. 258-269.

9. Ch a ndraka s a n, S. A. Dynamic Power Management in Wireless Sensor Network. - IEEE Design and Test of Computer, Vol. 18, June 2001, pp. 62-74.

10. E 1 s o n, J., D. E s tri n. Time Synchronization for Wireless Sensor Network. - In: Proc. of 15th Parallel and Distributed Processing Symposium, Vol. 14, July 2001, pp. 1698-1702.

11. Y a o, Y. C., Y. Y a o. The Application of Ant Colony Optimization in Wireless Sensor Network Routing. - Advanced Materials Research, Vol. 5, May 2013, pp. 838-841.

12. Z h a n g, J. Y., D. Y. C h e n. Clustering Routing Algorithm Ant Colony Optimization-Based for Wireless Sensor Network. - Applied Mechanics and Materials, Vol. 58, April 2015, pp. 591-597.

13. Z h o n g, J. H. Ant Colony Optimization Algorithm for Lifetime Maximization in Wireless Sensor Network with Mobile Sink. - In: Proc. of 14th International Conference on Genetic and Evolutionary Computation, 2012, GECCO’12, pp. 1199-1204.

14. L y n e t te, L. Using Artificial Intelligence to Optimize Wireless Sensor Network Deployments for Sub-Alpine Biogeochemical Process Studies. - In: 87th AMS Annual Meeting, 2007, pp. 230-236.

15. J u 1 i o, B., L. C ar lo s, M. J a vi e r. A New Wireless Sensor Network Routing Protocol Based on Artificial Intelligence. - Lecture Notes in Computer Science, Vol. 3842, August 2006, pp. 271-275.

16. Julio, B. Giving Neurons to Sensors: An Approach to QoS Management through Artificial Intelligence in Wireless Networks. - Lecture Notes in Computer Science, Vol. 4217, May 2006, pp. 344-355.

17. P eter, M. Model-Driven Design Plus Artificial Intelligence for Wireless Sensor Networks Software Development. - In: Proc. of International Conference on Software Engineering, 2011, pp. 63-64.

18. Luca, P., B. Antonino. Artificial Intelligence and Synchronization in Wireless Sensor Networks. - Journal of Networks, Vol. 4, August 2009, pp. 382-391.

19. J a n, N., K. R y s z a rd, N. Maci ej. Directed Communication in Wireless Sensor Network Based on Digital Terrain Model. - In: 2nd International Symposium on Logistics and Industrial Informatics, 2009, LINDI'09, pp. 231-236.

20. L on g, H. Q., G. Z. G u o. Research on Cloud Trust Model for Malicious Node Detection in Wireless Sensor Network. - Tien Tzu Hsueh Pao/Acta Electronica Sinica, Vol. 40, November 2012, pp. 2232-2238.

21. Celalettin, K. Energy and Lifetime Analysis of Compressed Wireless Sensor Network Communication. - In: Proc. of IEEE Sensors Applications Symposium, 2013, SAS'13, pp. 7-10.

22. N e h a, K. Mathematical Model on the Transmission of Worms in Wireless Sensor Network. Applied Mathematical Modelling, Vol. 37, March 2013, pp. 4103-4111.

23. Xi a o ju a n, C., D. K. Mi e s o. Modelling the Energy Cost of a Fully Operational Wireless Sensor Network. - Telecommunication Systems, Vol. 44, June 2010, pp. 3-15. 